\title{
Maternidad Transnacional, un desafío para las mujeres peruanas migrantes trabajadoras del barrio Meiggs ${ }^{1}$
}

\author{
Andrea Avaria ${ }^{2}$, Viviana Avendaño ${ }^{3}$, Javiera Rivera ${ }^{4}$, \\ Yerma Sepúlveda ${ }^{5}$
}

\section{RESUMEN}

El presente artículo vincula la temática de la maternidad y la migración. Las mujeres se incorporan al mundo laboral, cumpliendo roles productivos y también reproductivos. Actualmente las mujeres migran más que los hombres. La migración de la mujer-madre, en el ámbito familiar, modifica el ejercicio de la maternidad. La maternidad pasa de ser cotidiana figura física, a una presencia a pesar de la distancia. Entenderemos la prolongación de este vínculo como "maternidad transnacional". Este texto aborda los desafíos que deben enfrentar las mujeres-madres que migran al ejercer su maternidad a distancia. Nos referiremos a las formas en que se ejerce la maternidad, los afectos y experiencias relacionadas con estar lejos de los hijos/as. Por último, abordaremos el ejercicio de la intervención profesional en este ámbito desde el Trabajo Social.

Palabras clave: Maternidad transnacional, cadena de cuidado, familia transnacional, migración peruana.

\section{Maternidade Transnacional, um desafio para as mulheres peruanas migrantes trabalhadoras do bairro Meiggs}

\section{RESUMO}

O presente artigo vincula o tema da maternidade e a migração. As mulheres se incorporam no mundo laboral, cumprindo papéis produtivos e também reprodutivos. Atualmente as mulheres migram mais que os homens. A migração da mulher-mãe, no âmbito familiar, modifica o exercício da maternidade. A maternidade passa de ser uma cotidiana figura física, a uma

Artículo recibido: 20/04/2016. Artículo aprobado: 14/09/2016.

2 Chilena. Asistente Social. Académica e Investigadora Universidad Autónoma de Chile. PRIEM- Universidad Alberto Hurtado. Correo electrónico: andreaavaria@gmail.com

3 Chilena. Licenciada en Trabajo Social con mención en Gestión de Políticas Públicas, Universidad Autónoma de Chile. Correo electrónico: viviana.elisa.av@gmail.com

4 Chilena. Licenciada en Trabajo Social. Universidad Autónoma de Chile. Correo electrónico: javierariso@gmail.com

5 Chilena. Licenciada en Trabajo Social con mención en Gestión de Políticas Públicas, Universidad Autónoma de Chile. Correo electrónico: yerma.vanessa@gmail.com 
presença apesar da distância. Entenderemos a prolongação desse vinculo como "maternidade transnacional". Este escrito aborda os desafios que devem enfrentar as mulheres-mães que migram ao exercer sua maternidade à distância. Referiremos-nos às formas em que se exerce a maternidade, os afetos e as experiências relacionadas com estar longe dos(as) filhos(as). Finalmente, abordaremos ao exercício da intervenção profissional neste âmbito desde o Serviço Social.

Palavras-chave: maternidade transnacional, cadeia de cuidado, família transnacional, migração peruana.

\section{The challenges of exercising transnational motherhood for Peruvian women migrants}

\section{ABSTRACT}

This article, intersect the motherhood and migration issues. Currently women migrate more than men, joining the labor sphere, fulfilling productive and reproductive roles. The migration of mothers causes consequences to her, like to modify their exercise of motherhood, from the physical and daily presence, to a distance presence: "transnational motherhood". Therefore, the following article talks about the challenges facing these mothers to exercise their maternity despite the physical distance, we will describe the ways that their maternity is exercised and the feeling that came from be away from their children. Finally, we reflect on how social work can intervene at the migration scenario.

Keywords: Transnational motherhood, care chains, transnational family, peruvian migration.

\section{Antecedentes}

Actualmente la migración interregional ha aumentado producto del dinamismo que trae la globalización, esta ha movilizado diversos flujos, tanto de capitales, como de recursos y de personas (Martínez, Cano \& Soffia, 2014). La migración interregional, se produce fundamentalmente entre países limítrofes en el continente americano, permitiendo que las personas tengan mayor movilidad espacial y con ello laboral. La población peruana en Chile, como ejemplo de ello, constituye la corriente migratoria que lidera en número, con un $31,7 \%$ de población migrada, seguido de los argentinos con un $16,3 \%$ y los bolivianos con un $8,8 \%$, de acuerdo a datos del Departamento de Extranjería y Migración (DEM, 2016). 
La migración es principalmente laboral. La población que ingresa a Chile se encuentra, fundamentalmente, entre los 20 y 35 años, edad económicamente activa (DEM, 2016). Según la Comisión Económica para América Latina y el Caribe (Martínez, Cano \& Soffia, 2014), la migración femenina ha ido aumentando gradualmente en países de la región latinoamericana, lo que se relaciona con la inserción de la mujer en el ámbito laboral. Al respecto, el DEM (2016) refiere que existe un 53,5\% de mujeres migrantes en Chile frente al 46,5\% de hombres que migran.

Stefoni (2011) afirma que las mujeres migrantes emprenden el viaje al país de destino de manera solitaria, debido a la incertidumbre de no contar con trabajo o lugar donde vivir, situación que sería más compleja si vinieran con sus hijos/as.

Debido al progresivo aumento de la migración femenina, en el contexto de la globalización, ta mujer que es madre y migrante, ha tenido que dejar el cuidado de sus hijos/as a un tercero, por lo general mujer, lo que conocemos como "cadenas globales de cuidado" (Pérez, 2007). La migración de las mujeres ha complejizado la valoración que socialmente tenemos de las mujeres, especialmente de las mujeres-madres, tanto en las sociedades de origen y destino, pues representamos a estas mujeres como "malas madres", puesto que con la migración las mujeres abandonarían sus hogares y a sus familias; sin embargo, ya sabemos que la migración no implica el cese del ejercicio de la maternidad (Pérez, 2007). Gregorio (2011) se refiere a la maternidad transnacional, como la relación que las mujeres mantienen con los hijos a pesar de la distancia física con ellos/as, prolongando y reformulando funciones y roles que en los países de destino ellas ya asumían.

Los sentimientos asociados al ejercicio de la maternidad a distancia, está cargado muchas de las veces con culpas, las que emergen a través del cuestionamiento que en las sociedades (de origen y de destino) realizamos a las mujeres, por el hecho de alejarse de sus familias y de sus hogares. Al mismo tiempo, para las mujeres, el hecho del trabajo y el desarrollo de una vida laboral, está asocia- 
do a sentimientos de orgullo y satisfacción, pues a través de sus trabajos proporcionan una mejor calidad de vida a sus familias (Gregorio, 2011).

El ejercicio de la maternidad transnacional se hace posible por medio de las "cadenas globales de cuidado" (Pérez, 2007), redes que por lo general se construyen con la participación de otras mujeres del entorno familiar o social de las mujeres migrantes. El uso de las "Tecnologías de la Información y la Comunicación" (TIC) ha favorecido, por cierto, la oportunidad de comunicación inmediata y democrática.

El "Observatorio Iberoamericano sobre Movilidad Humana. Migraciones y desarrollo”, estima que para el 2014 residían en Chile unas 411.000 personas provenientes de otros países, lo que corresponde a un 2,3\% de la población nacional; la mayoría (tres cuartas partes) corresponde a población sudamericana que ingresa a Chile (Rojas \& Silva, 2016). La población migrada, se concentra en la Región Metropolitana (61,5\%) según datos para el 2014, constituyendo un 3,5\% de la población habitante de la región (Rojas \& Silva, 2016). Según estos datos, la principal población residente en Chile es "la de los peruanos (31,7\%), seguidos por los argentinos (16,3\%), bolivianos $(8,8 \%)$, colombianos $(6,1 \%)$ y ecuatorianos $(4,7 \%)$ " (Rojas \& Silva, 2016, p. 14). Distintas investigaciones dan cuenta de una feminización de la migración contemporánea (Martínez, Cano \& Soffia, 2014; Stefoni, 2011, 2002; Cano, Soffia \& Martínez, 2009).

En la Región Metropolitana la migración se distribuye en algunas comunas, en particular, en Estación Central la población migrada es de 1.354 habitantes peruanos (CENSO 2002 en Stefoni, 2004). Respecto al ámbito laboral de quienes migran, es posible afirmar que la población peruana se inserta en nichos particulares, pues para ellos se ofrecen trabajos precarios: por lo general, las mujeres se insertan en servicios domésticos y también en sectores del comercio, los hombres lo hacen en la construcción y el comercio o servicios; según Stefoni (2011) el 16\% de los peruanos se desempeña en servicios y como vendedores de comercio y mercado. 
En este contexto, para comprender la maternidad de las mujeres madres migrantes peruanas resultó importante concentrarnos en mujeres que sólo ejercieran el rol de madres y no de cuidadoras (asesoras de hogar), es decir, la investigación se constituye en una oportunidad para recoger las experiencias de las mujeres que trabajan en el área del comercio, que tuvieran hijos e hijas en origen, puesto que sus experiencias darían cuenta de la maternidad a distancia.

Para efectos de este artículo, solo se hará referencia a las cadenas de cuidado y a los sentimientos que provoca en las madres ejercer maternidad transnacional (la investigación mayor implicó dar cuenta del uso y frecuencia de tecnología en el ejercicio de la maternidad a distancia).

\section{Ser mujer, madre y migrante, las tensiones en el imaginario cultural de la sociedad}

La migración femenina ha sido explicada desde diversas aproximaciones teóricas. Pedone (2008) ha señalado que el género es una condicionante que influye en la migración. Cuando es el hombre quien migra, se asume que es el jefe de hogar y va en búsqueda del sustento económico para su familia; con ello se produce un reforzamiento del rol de proveedor económico, recibiendo por ello reconocimiento social.

Por el contrario, cuando es la mujer quien migra, se produce un fenómeno en particular, las mujeres rompen con el rol reproductor que le ha conferido la sociedad, y pasan a ejercer un rol asociado a la producción económica (Pedone 2004; Gregorio, 2011). Las mujeres en este marco se relacionan con sus familias a través del envío de remesas (Pedone, 2004). Sin embargo, este hecho (migración y trabajo) no es evaluado positivamente por la sociedad, pues históricamente se define a las mujeres en relación a una esfera privada, social y reproductiva, mientras que el hombre es asignado a la esfera pública, económica y productiva (Gregorio, 1997).

En este contexto migratorio se produce un fenómeno de desnaturalización, o cuestionamiento de estos supuestos, se deja de con- 
siderar como natural "la relación "mujer $=$ madre $=$ cuidadora" como un hecho dado (...)” (Gregorio, 2012, p. 586). Con la migración de las mujeres-madres, el rol reproductivo que apunta al ejercicio de la maternidad, se modifica para dar paso a la incorporación de las mujeres a la esfera laboral y social, es decir, las mujeres asumen un rol productivo, sin abandonar el rol reproductivo asignado socialmente. La dicotomía entre lo privado y lo público,no permite visibilizar las complejidades del fenómeno de la maternidad transnacional, puesto que las mujeres desempeñan ambos roles, pudiendo renegociar relaciones de poder y género más equitativas (Gregorio, 2011).

\section{El cuidado, un rol de las mujeres}

El contexto migratorio supone la separación entre la mujer que migró y la familia que permanece en el país de origen; este hecho configura un nuevo tipo de familia, las denominadas familias transnacionales (Stefoni, 2002). Estas familias mantienen los lazos familiares, vínculos de afectos y confianzas en un espacio físico no cohabitado, manteniendo la relación más allá de las fronteras ( $\mathrm{Pa}-$ rella, 2007; Valdebenito, 2010).

La familia transnacional (Parella, 2012; Stefoni, 2002) cumple un rol importante para apoyar a la mujer-madre transnacional y sus hijos/as que permanecen en el país de origen; esta se encarga de las complejidades que pueda traer el proceso migratorio (León, 2013). Previo a la migración, la mujer-madre deja el cuidado de sus hijos/as a otra mujer, que por lo general pertenece a su familia nuclear o extensa. Este hecho se conoce como "cadenas globales de cuidado" (Herrera \& Carrillo, 2009; Pérez, 2007), y "se conforman con el objetivo de sostener cotidianamente la vida, y en las que en los hogares se transfieren trabajos de cuidados de unos a otros en base a ejes de poder, entre los que cabe destacar el género (...)" (Pérez, 2007, p. 4). Tal como refieren Herrera \& Carillo (2009), el cuidado se reorganiza entre las abuelas, tías, etc., y la cuidadora cumple el papel que desempeñaba la madre cuando estaba físicamente presente. 
Son pocas las mujeres que dejarían el cuidado de sus hijos/as a los padres. Aun así, hay casos en que los hombres participan de las prácticas de cuidado, sin embargo, lo harían asumiendo un rol secundario, ya que su labor estaría apoyada de forma constante, por otra mujer (Arriagada \& Todaro, 2012; Pérez, 2007).

Nuevamente entra en juego una visión tradicional del cuidado como una actividad a cargo de las mujeres, visión que es reproducida por las propias madres migrantes, quienes se sienten en algunos casos más seguras si sus hijos quedan a cargo de los abuelos en vez de con sus parejas (...) (Herrera \& Carrillo, 2009, p. 6).

La "cadena de cuidado" es uno de los facilitadores que permite a la mujer-madre ejercer maternidad transnacional, realizando este cuidado por medio de remesas sociales y económicas.

Las remesas sociales se materializan a través de llamadas telefónicas, envío de regalos y visitas esporádicas al país de origen, es decir, encargarse "del cuidado y crianza de los hijos, a pesar de la distancia" (León, 2013, p. 23); de esta manera se actualiza el lazo afectivo. Por otro lado, el envío de dinero permite satisfacer las necesidades económicas de los hijos/as y la familia que permanecen en el país de origen (Zapata, 2009).

Las mujeres que migran siguen participando en la vida cotidiana de sus hijos/as, "les ayudan con las tareas del colegio, los cuidan si están enfermos, se preocupan de ellos en todos los aspectos de sus vidas. Ya sea a distancia o no, la mujer se hace cargo de la crianza" (León, 2013, p. 24).

\section{Los sentimientos que genera ejercer "Maternidad Transnacional"}

Para las mujeres, realizar el proyecto migratorio es complejo, por el hecho de tener que dejar a sus hijos/as en el país de origen; con frecuencia emerge un cuestionamiento al sentido del proyecto migratorio. Al respecto, Ariza (2011) afirma que las mujeres experimentan sentimientos de tristeza, expresada en llanto, falta 
de apetito, la sensación de haber abandonado a sus hijos/as; estos aspectos se reúnen en un sentimiento de culpa constante. Paralelamente, sin embargo, experimentan orgullo y satisfacción por contribuir a mejorar la situación económica de sus familias en origen. Las mujeres, también, se sienten empoderadas, debido a que reciben ingresos producto de las relaciones laborales de comercio en las que se insertan, y ello impacta en un aumento de la autoestima.

El proyecto migratorio en las mujeres despierta sentimientos ambivalentes. En ellas se evidencia la contradicción de la migración; por un lado, migran para dar mayor bienestar a sus hijos/as, pero, por otro, se producen costos asociados a la ausencia física de la figura materna, afectando a todas las personas involucradas (Ariza, 2011).

\section{Metodología}

La investigación se realizó bajo la metodología cualitativa (Krause, 1995), desde un enfoque fenomenológico, que permitió aproximarnos a los sentidos y significados de las experiencias de las mujeres-madres peruanas. A través de este estudio de carácter descriptivo, pudimos profundizar en la experiencia subjetiva de la maternidad a distancia, especialmente de las mujeres peruanas que trabajan en el comercio, en el sector del Barrio Meiggs, de Estación Central.

Concentrarse en mujeres migradas trabajadoras en áreas de comercio, permitió acercarse a mujeres que, en su condición de trabajadoras, se insertan en labores asalariadas fuera del ámbito de los servicios domésticos (roles reproductivos), nicho laboral que desde la sociedad chilena se suele asignar a las mujeres provenientes de Perú (cuidados y trabajo doméstico), reduciendo con ello y para ellas, las oportunidades laborales en otros campos de ocupación (alejados de lo doméstico).

La comprensión de las experiencias de las mujeres-madres peruanas se realizó a través de la entrevista en profundidad (Mejía, 
1999, citado por Quintana, 2006); para ello se realizaron 10 entrevistas, con la finalidad de identificar la dinámica en la "cadena de cuidado" de la que forman parte las mujeres-madres migrantes y comprender, además, los sentimientos que provoca la maternidad transnacional ${ }^{6}$.

Se contactó y seleccionó a 10 mujeres madres peruanas entre los 19 y 47 años ${ }^{7}$, con hijos/as menores de 18 años, que estuvieran en sus lugares de origen. Hijos/as que, en Perú, estuviesen a cargo de otra mujer perteneciente a la familia extensa de la madre o del padre. Mujeres que vivan al menos un mes en Chile ${ }^{8}$. Mujeres que se desempeñaran en trabajos en el ámbito del comercio, en el sector del Barrio Meiggs, por ser este un espacio de comercio de recepción histórica de migrantes. De esta manera se constituyó la muestra, a través de un muestreo intencional opinático (Ruiz Olabuénaga, 2012).

\section{Resultados}

\section{La "cadena de cuidado" como un facilitador de la "maternidad transnacional"}

La mujer-madre migrante en Chile, por lo general, se convierte en la principal sostenedora económica de los hijos/as y de la familia que permanece en Perú. Los cuidados que ejercen las mujeresmadres desde Chile a Perú y que son constitutivos de la dinámica de las cadenas de cuidado y del ejercicio de la maternidad a distancia, forman parte de los hallazgos significativos en el marco de la investigación.

6 La investigación en su totalidad consideró además la exploración respecto de los usos de las tecnologías en el mantenimiento de las redes de colaboración, las "cadenas de cuidado" y el ejercicio de la "maternidad transnacional". Este artículo da cuenta de parte de los resultados.

7 Del total de 10 mujeres: 2 de ellas tenían 19 años (1 hijo cada una); 1 mujer de 28 años (1 hija de 9 años); 1 mujer de 29 años (2 hijas); 1 mujer de 32 años (1 hijo); 1 mujer de 39 años (1 hijo de 11 meses); 1 mujer de 40 años (hijos de 14, 18, 20); 1 mujer de 42 años (2 hijos de 17 y 21, 1 hija de 23); 1 mujer de 45 años (3 hijos de 4, 16, 25 y 1 hija de 23); 1 mujer de 47 años (1 hijo de 15 años).

8 Un mes a considerar desde agosto 2015. 
Los cuidados de las mujeres-madres en el contexto migratorio, se producen a distancia. Estos persisten y se ejercen a través de remesas sociales y económicas. Las mujeres-madres entrevistadas envían remesas monetarias para costear, principalmente la educación de sus hijos/as, gastos de alimentación, y remesas sociales como un mecanismo para expresar sus afectos y constante preocupación a pesar de la ausencia física (Zapata, 2009).

"[...] Y mi labor estando lejos es preocuparme de mandar el dinero, para pagar la pensión del colegio, para la comida, [...] dentro de lo poco que tengo, darle todo lo que yo pueda [...]" (María, octubre 2015).

La mayoría de las mujeres-madres entrevistadas dejaron a sus hijos/as a cargo de otra mujer, por lo general parte de la familia extensa, a pesar de que los niños/as cuentan con padres biológicos, estas mujeres-madres refieren desconfiar de los cuidados que los hombres (progenitores) pueden dar a los hijos/as, apuntando a la falta de compromiso, tiempo y dedicación a los cuidados.

“Nooo, al papá no, no la puedo dejar al papá (ríe) [...], no tengo mucha confianza en él [...] de repente él ha trabajado, él está siempre más alejado, el papá trabaja y la deja y se va [...]" (Yeni, septiembre 2015).

A pesar de esto, 3 mujeres-madres entrevistadas dejaron a los hijos/as al cuidado de sus padres. Estos hijos/as, sin embargo, son adolescentes y gozan de más independencia; por lo tanto, el padre cumple un rol más distante en los cuidados. Los relatos coinciden en afirmar que al lado de un hombre hay una mujer que les ayuda en las tareas domésticas (Pérez, 2007). La presencia de algunos padres en la "cadena de cuidado", constituye un hallazgo relevante:

“[...] quedamos que él se quedara [...] y como yo me vine acá, ellos se quedaron ahí con el papá. Era también mucho mejor porque a las finales es su papá, entonces mejor que se quedaran ahí [...] y no hubo problema que ella (la cuñada) le cocinara y eso [...]" (Naty, septiembre 2015). 
Wagner (2008) afirma que las mujeres migran solas. En el contexto de la investigación se observó, sin embargo, que 4 de las 10 entrevistadas han migrado en compañía de sus parejas. Estas parejas no en todos los casos fueron los padres biológicos de los niños/as, en las familias en que son los progenitores de los hijos/as, ambos se ocupan de los cuidados, es decir, tanto la madre como el padre, se comunican con los hijos/as que permanecen en Perú a través de los medios tecnológicos.

"Bueno, le pregunta cómo estás hijita, este te queremos, que estamos bien, que ya estaremos ahí para pasear y ella le dice ya, bueno, bueno" (Julia, septiembre 2015).

Respecto de la mujer cuidadora de los hijos/as en Perú, es relevante señalar que ninguna de las mujeres entrevistadas sostuvo acuerdos formales, contrato u otro, que detallara tareas y responsabilidades de cuidado u obligaciones con las mujeres-madres migrantes. Ambas mujeres se comunican directamente y establecen los acuerdos de palabra en el marco del cuidado en el contexto de migración de la madre; esta comunicación es directa, fluida y constante.

En origen, las mujeres-cuidadoras asumen funciones económicas, reciben y administran el dinero que envían las mujeres-madres a Perú, y con ello satisfacen las necesidades de los y las hijas.

Los cuidados diarios que ejercen las cuidadoras hacia los hijos/ as, varían de acuerdo a la edad de los niños/as. Cuando los hijos/ as son pequeños, la mujer-cuidadora se encarga de las mismas labores que realizaba la mujer-madre transnacional cuando vivía en Perú, tales como: limpiar la casa, cocinar, ayudar a los hijos/as. En los casos en que los hijos/as son adolescentes, la mujer-cuidadora se encarga principalmente de supervisar el desempeño escolar de estos.

\section{La ambivalencia de sentimientos en el ejercicio de la "maternidad transnacional"}

Las entrevistadas refieren experimentar sentimientos de tristeza y nostalgia, producto de la lejanía de los hijos/as; estos sentimientos 
se relacionan también, con estar lejos de la familia y de la cultura peruana, la lejanía del origen.

“[...] pero más que todo yo siento pena, nostalgia, no solo de mi hijo, sino de la familia, de mi tierra, mis costumbres, mi comida, es todo ajeno, [...] y esa es la pena que uno tiene de dejar su tierra, la familia y venir tan lejos. Yo creo que ese sentimiento lo tenemos todos los que hemos venido acá" (María, octubre 2015).

Todas las mujeres entrevistadas coinciden en experimentar sentimientos ambivalentes. Por un lado, mantienen comunicación directa y cotidiana con sus hijos/as a través de los medios tecnológicos, la acción de enviar remesas las hace sentir aliviadas, sin embargo; la distancia física con sus hijos/as les afecta, manifestándose a través de sentimientos de tristeza y culpa por dejarlos/las. Por otro lado, sienten orgullo y satisfacción pues su trabajo y el hecho de migrar, permite que sus hijos/as mejoren su calidad de vida.

Es importante señalar que, para las mujeres-madres transnacionales, la emancipación producto de la migración es parcial, en tanto las familias en Perú son dependientes del trabajo que estas realicen, a través de las remesas económicas (Gregorio, 2011).

Para las mujeres entrevistadas, el ejercicio de la maternidad transnacional evidencia que la maternidad es un vínculo que perdura pese a la distancia entre ellas y sus hijos/as.

\section{Reflexiones finales}

La complejidad de la migración femenina, en particular de las mujeres-madres, pone de manifiesto nuevos desafíos; debemos cuestionar el qué hacer, y el modo de entender lo social; como Trabajadores Sociales debemos enfrentar, tanto en lo profesional, como en lo personal, cuestionamientos, debido a la complejidad que experimenta la sociedad contemporánea, respecto a cuestiones de género y migración. Las mujeres peruanas que vienen a Chile sin sus hijos/ as, encaran dificultades en su proyecto migratorio. Principalmente porque son juzgadas socialmente, a través de consideraciones sub- 
jetivas, la sociedad cuestiona el rol materno de las mujeres, puesto que dejar a los hijos/as en origen es interpretado como abandono, $y$, por tanto, una falta en su rol de madre, y con ello se objeta su condición de mujer. Nuestra labor ético-profesional es estar atentos/as a la reproducción, en la intervención, de estos juicios y prácticas que no contribuyen a la inclusión social de los sujetos.

Las experiencias recogidas durante la investigación muestran que las mujeres cumplen una dualidad de roles, por un lado, mantienen su rol reproductivo, pues las mujeres siguen presentes en la vida de los hijos/as a pesar de la distancia geográfica, a través de prácticas transnacionales, tales como el envío de remesas sociales y económicas; y, por otro lado, cumplen un rol productivo al insertarse al mundo laboral en las sociedades de destino.

Las mujeres se cuestionan la maternidad y experimentan culpa por no estar físicamente con sus hijos/as. Al mismo tiempo, sienten orgullo de haber emprendido la migración, puesto que, a través del trabajo y el envío constante de remesas económicas, aseguran a los hijos/as una mejor calidad de vida. La tensión entre la valoración social y cultural de las mujeres en su condición de madres y la experiencia de las mujeres trabajadoras y migrantes, evidencia las complejidades de ser madre transnacional (Gregorio, 2011; Parella, 2012).

El hecho de que estas mujeres asuman un rol productivo en la sociedad, y continúen en el ejercicio de un rol reproductivo, se hace posible pues otras mujeres forman parte de la "cadena de cuidado", compuesta por lo general, por eslabones femeninos en los lugares de origen.

En este contexto, desde el Trabajo Social, es fundamental, por una parte, elaborar estrategias de cuidado de los derechos de las mujeres en su condición de trabajadoras en destino, y, por otra parte, propender a la participación de los varones en prácticas de cuidado de las familias, a fin de cuestionar el ejercicio de los roles de hombres y mujeres, propendiendo al equilibrio en el ejercicio de los roles de género (Gregorio, 2012). 
La investigación ha permitido comprender la complejidad de las relaciones de género respecto a la maternidad a distancia. Da cuenta de la necesidad de reflexionar como sociedad de destino, desde las aproximaciones cotidianas y profesionales que realizamos hacia las mujeres y el ejercicio de su maternidad. Para mejorar esta comprensión, se hace indispensable aproximarnos, sin duda, a las experiencias de los hijos/as que quedan en el país de origen y los padres.

Tal como mencionan Aguilar \& Buraschi (2014), "como consecuencia de la globalización, los trabajadores sociales estamos inmersos en procesos constantes de cambio, y el hecho migratorio, junto con el desafío intercultural que implica, nos impone la necesidad de un reciclaje y una formación continua [...]" (p. 278), y la maternidad transnacional nos invita a "repensar nuestra forma de concebir la identidad, la cultura, la participación [...]" (Aguilar \& Buraschi, 2014, p. 279). Como profesionales debemos cuestionar construcciones y representaciones sociales de familia, parentalidad-maternidad, concepciones y principalmente prácticas de intervención social, pues como profesionales nos encontramos en contextos de relaciones transnacionales y de relaciones de poder que debemos considerar, tanto en el ejercicio de intervención como también de investigación (Avaria, 2012).

El desarrollo de las comunicaciones y la tecnología permite el sostenimiento de los vínculos afectivos, por ende, la ausencia física "no necesariamente se debe interpretar como indicador de fragmentación familiar; migrar no implica taxativamente precarizar los vínculos familiares" (Pombo, 2012, p. 17). Estas nuevas realidades, y las interpretaciones más amplias deben tener un correlato en las prácticas de intervención social, pues estas deben incorporar una reflexión respecto de la episteme de los sujetos y de lo social desde el trabajo social; los nuevos contextos nos interpelan para cuestionar y transformar las prácticas sociales, y debemos reconocer que estas prácticas tienen un correlato en las prácticas asistenciales y en las políticas sociales (Avaria, 2007). 
En este marco migratorio, se hace necesario problematizar y revisar las políticas públicas ya que responden a lógicas hegemónicas y rígidas que no consideran la transnacionalidad de los sujetos y sus relaciones sociales; debemos "garantizar derechos de quienes integran estas familias, en particular de los/as niños/as y adolescentes, y de las mujeres migrantes" (Pombo, 2012, p. 17).

Como Trabajadores Sociales debemos ser ejes transformadores para intervenir con familias transnacionales y sus entornos (Parella, 2012; Stefoni, 2002), utilizando estrategias propias del modelo intercultural como el empoderamiento para "reducir, eliminar, combatir o invertir las valoraciones negativas que se plantean, desde el conjunto de la sociedad en general y desde el poder y sus grupos en particular, sobre los inmigrantes" (Aguilar \& Buraschi, 2014, p. 282), fortaleciendo las redes de apoyo, habilidades, tecnologías y capacidad para tomar decisiones, debemos ser conscientes del lugar político que nos corresponde en el ámbito de la acción con personas migrantes (Aguilar, 2012; Avaria, 2012).

Es un llamado a permanecer atentos/as a las lógicas culturalistas o etnocéntricas, que por lo general se subsumen en las intervenciones o en las evaluaciones que como profesionales hacemos de la realidad, o de las interpretaciones de los éxitos o fracasos que realizamos de nuestras intervenciones profesionales (Aguilar, 2012; Avaria, 2012).

Debemos aproximarnos y comprender a las mujeres. La migración en muchos de los casos, se constituye en una acción emancipadora para ellas, sin embargo, esta no necesariamente transforma sus realidades, dado el rol de proveedoras que asumen (Gregorio, 2012, 2011).

Fuentes (2014) afirma que se requiere coordinación y apoyo institucional más allá de las fronteras, "transformando las prácticas institucionales e incorporando recursos comunicativos (internet, teléfono, video llamada, etc.) entre organizaciones que fomenten el transvase y la información" (p. 93) sobre el caso que se intervie- 
ne, superando los alcances que tienen las políticas del Estado/Nación, para intervenir en el contexto transnacional.

La compleja realidad de la migración, y de los sujetos que la viven, es un desafío para quienes realizan trabajo social, el cuestionamiento a las bases sociales y teóricas en que hemos sido formados implica un reto, nos abre a nuevos cuestionamientos; es una oportunidad para transformar modos de intervención, tanto desde las políticas sociales, como desde las instituciones, que, sin duda, podrán mejorar el modo en que realizamos nuestro aporte como disciplina para la sociedad en su conjunto.

\section{Bibliografía}

Ariza, M. (2011). Vida familiar transnacional en inmigrantes de México y República Dominicana en dos contextos de recepción. Si Somos Americanos. Revista de Estudios Fronterizos, 12(1), $17-47$.

Aguilar, M.; Buraschi, D. (2014). Formación en Trabajo Social con conciencia global y compromiso local: un caso de buena práctica educativa. [Versión Electrónica]. Cuadernos de Trabajo Social, 27 (2), 277- 289.

Aguilar, M. (2012). Pensar la intervención social con personas migradas: un desafío a nuevas formas de intervención desde el trabajo social. En Avaria, A. Desafíos de la migración. ¿Cómo acercarnos a las personas migradas? Miradas de y desde la investigación e intervención social (p. 79-111). Santiago de Chile. Ediciones Universidad Santo Tomás.

Arriagada, I. \& Todaro, R. (2012). Cadenas globales de cuidado: El papel de las migrantes peruanas en la provisión de cuidados en Chile. ONU Mujeres. Santiago de Chile.

Avaria, A. (2012). Desafíos de la migración. ¿Cómo acercarnos a las personas migradas? Miradas de y desde la investigación e intervención social. Santiago de Chile. Ediciones Universidad Santo Tomás.

Avaria, A. (2007). El Estado y la incorporación de las diferencias. ¿Problema resuelto? En Fundación CIDOB. Pensar las dinámicas interculturales. Aproximaciones y perspectivas. Foro de doctorandos. Documentos CIDOB. № 10. Dinámicas interculturales. (P. 122- 140). Barcelona.

Cano, M.; Soffia, M. \& Martínez, J. (2009). Conocer para legislar y hacer política: los desafíos de Chile ante un nuevo escenario mi- 
gratorio. Serie Población y Desarrollo, 88. CEPAL (Comisión Económica para América Latina y el Caribe).

DEM (Departamento de Extranjería y Migración) (2016). Anuario Estadístico Nacional 2005-2014. Recuperado el 10 de septiembre 2016 de http://www.extranjeria.gob.cl/media/2016/02/Anuario-Estad\%C3\%ADstico-NacionalMigraci\%C3\%B3n-en-Chile-2005-2014.pdf

Fuentes, V. (2014). Intervención Social Local con mirada global. La propuesta de Trabajo Social Transnacional con familias (in)migrantes y/o transnacionales entre Bolivia y España. Portularia, 14(1), pp. 87-95. Recuperado el 13 de septiembre 2016 de: http://www.redalyc.org/articulo. oa?id=161029523008

Gregorio, C. (1997). El estudio de las migraciones internacionales desde una perspectiva de género. Migraciones (1), 145-175. Recuperado 13 septiembre 2016 en: http://www.ciudaddemujeres.com/articulos/IMG/pdf/MIGRACIONES-CarmenGregorio.pdf

Gregorio, C. (2011). Análisis de las migraciones transnacionales en el contexto español, revisitando la categoría de género desde una perspectiva etnográfica y feminista. [Versión Electrónica]. Nueva antropología, 24(74), 2-20.

Gregorio, C. (2012). Tensiones conceptuales en la relación entre género y migraciones. Reflexiones desde la etnografía y la crítica feminista. [Versión Electrónica]. Papers, 97 (3), 569- 590.

Herrera, G.; Carrillo, M. (2009). Transformaciones familiares en la experiencia migratoria ecuatoriana. Una mirada desde los contextos de salida. [Versión Electrónica]. Mélanges de la Casa de Velázquez, 39(1), 1-15.

Krause, M. (1995). La investigación cualitativa: Un campo de posibilidades y desafíos. Revista Temas de Educación (7), 19-39.

León, C. (2013). La retradicionalización de los roles de género en la maternidad transnacional: el caso de mujeres peruanas en Santiago de Chile. Si Somos Americanos Revista de Estudios Fronterizos, 14(1), 15-40. Recuperado el 13 de septiembre 2016 de http://www.scielo.cl/pdf/ssa/v14n1/art02.pdf

Martínez, J.; Cano, M. \& Soffia, M. (2014). Tendencias y patrones de la migración latinoamericana y caribeña hacia 2010 y desafíos para una agenda regional. Serie población y desarrollo, 109. CEPAL (Comisión Económica para América Latina y el Caribe). 
Maternidad Transnacional, un desafío para las mujeres peruanas migrantes trabajadoras del barrio Meiggs / Avaria et al.

Parella, S. (2007). Los vínculos afectivos y de cuidado en las familias transnacionales. Migrantes ecuatorianos y peruanos en España. Migraciones internacionales, 2, 151-158.

Parella, S. (2012). Familia transnacional y redefinición de los roles de género. El caso de la migración boliviana en España. Papers, (97), 661-684.

Pedone, C. (2004). Negociaciones en torno al asentamiento definitivo de las familias migrantes ecuatorianas: Construcción de espacios sociales transnacionales. Ponencia presentada en el $4^{\circ}$ Congreso sobre la Inmigración en España (Girona, noviembre, 2004).

Pedone, C. (2008). "Varones aventureros" vs. "Madres que abandonan": reconstrucción de las relaciones familiares a partir de la migración ecuatoriana. [Versión Electrónica]. REMHU. Revista Interdisciplinar da Movilidade Humana, 16(30), 45-64.

Pérez, A. (2007). Cadenas globales de cuidado. Serie Género Migración y Desarrollo. UN-INSTRAW. (2). p. 1-9. Recuperado el 13 de septiembre 2016 de: http://mueveteporlaigualdad.org/publicaciones/cadenasglobalesdecuidado_orozco.pdf

Pombo, M. (2012). A propósito de la intervención con familias transnacionales. O la intervención como frontera. Savia, 11(10), p. 11 - 19. Recuperado el 13 de septiembre 2016 de: http:// www.trabajosocial.uson.mx/imagenes/savia10.pdf

Quintana, A. (2006). Metodología de investigación científica cualitativa en Quintana, A. y Montgomery, W. (Eds.) Psicología: Tópicos de actualidad, Lima, UNMSM. 2006. pp. 47-84. Recuperado el 10 septiembre 2016, de http://cienciassociales. webcindario.com/PDF/Cualitativa/Inv_quintana.pdf

Rojas, N.; Silva, C. (2016). Informe OBIMID: La migración en Chile: Breve reporte y caracterización. Madrid. OBIMID. Recuperado el 10 septiembre 2016, de: http://www.extranjeria. gob.cl/media/2016/08/informe_julio_agosto_2016.pdf

Ruiz Olabuénaga, J. (2012). Metodología de la investigación cualitativa. España: Universidad de Deusto.

Stefoni, C. (2002). Mujeres inmigrantes peruanas en Chile. Papeles de Población, 8(33), julio-septiembre. 118-145. Recuperado 10 septiembre 2016 de: http://www.redalyc.org/articulo. oa?id=11203304

Stefoni, C. (2004). Inmigración y ciudadanía: la formación de comunidades peruanas en Santiago y la emergencia de nuevos ciudadanos. Política, (43), 319-336. Recuperado 10 septiembre 2016 de: http://www.redalyc.org/articulo.oa?id=64504313 
Stefoni, C. (2011). Perfil migratorio de Chile. OIM. Buenos Aires. Recuperado 10 septiembre 2016 de: http://priem.cl/wp-content/uploads/2015/04/Stefoni_Perfil-Migratorio-de-Chile.pdf

Valdebenito, X. (2010). Lima \$40/ Santiago \$50: ¿Amigo, tiene una cabina? Los inmigrantes y las TIC: la formación de la familia transnacional peruana (Tesis de pregrado). Universidad de Chile, Santiago de Chile.

Wagner, H. (2008). Maternidad transnacional: discursos, estereotipos y prácticas. En Herrera, G; Ramírez, J (eds.) América Latina Migrante: estado, familias, identidades. Quito, Flacso-Sede Ecuador: Ministerios de cultura del Ecuador. pp. 325-340. Recuperado el 13 de septiembre 2016 de: http://www.flacsoandes.edu.ec/agora/maternidad-transnacional-discursos-estereotipos-practicas

Zapata, A. (2009). Familia transnacional y remesas: Padres y madres migrantes. [Versión electrónica]. Revista latinoamericana de ciencias sociales, niñez y juventud, 7(2), 1749-1769. 